\title{
Calculation of Statistics Relating to Periodic Variation
}

\author{
J. J. Howarth \\ Giotto Loess Research Group, Geography Department, Leicester University, LE1 7RH, UK \\ Email: Johnhowart@aol.com
}

Received 9 April 2014; revised 19 May 2014; accepted 7 July 2014

Copyright (C) 2014 by author and OALib.

This work is licensed under the Creative Commons Attribution International License (CC BY). http://creativecommons.org/licenses/by/4.0/

(C) (i) Open Access

\begin{abstract}
It is often necessary for astronomers to interpret observational data in terms of periodic variation, or an additive combination of periodic variations. This paper shows how statistics relating to periodic variation - the frequency, amplitude and phase - may be inferred from raw observational data and, just as importantly, it provides estimates for the accuracy of such statistics in terms of the observational error. Formulae are given to enable the extraction of these statistics, together with error bounds that apply. Their use is illustrated by synthetically derived data which also serve to demonstrate, by Monte-Carlo methods, the veracity of these formulae.
\end{abstract}

\section{Keywords}

Periodic Variation, Monte-Carlo, Statistical Estimation, Fourier Analysis, Variable Stars, Rotating Asteroids, Double Star Systems, Superhumps

Subject Areas: Applied Statistical, Mathematics, Classical Mechanics

\section{Introduction}

The photometric and astrometric observations of many astronomical systems display a periodic component; examples include pulsating variable stars, rotating asteroids, double star systems, superhumps in the light curves of dwarf novae. In order to be able to draw conclusions about such systems it is of particular importance to deduce the frequency (or period) of the oscillation, together with an estimate for the accuracy of the derived frequency. The shape of the light curve is generally important too; however, before the shape may be inferred, it is always necessary to know the period over which the observational data are to be folded. In some instances the oscillation may be expressed in the colour of the light rather than the brightness-for example, the movement of a spectral peak due to Doppler shift, and in yet others as an object's position in the sky, but the principles involved in deducing periodicity are essentially the same. Besides the frequency (or period) of the oscillation, the amplitude (from highest to lowest value) and phase (related to a point in time) are often of interest, and formulae 
for these will be derived too, along with corresponding indications of accuracy.

It is important, however, to note that these accuracies refer to the accuracy of the calculated statistics, not to the significance of the periodicity itself. The question of whether an imputed period is significant, or just a random manifestation of noise in the observations, is not considered here; the reader is referred to Howarth \& Greaves [1] for a formula for the significance of a period, based upon Koen [2].

Given $\mathrm{n}$ measurements $\mathrm{m}_{\mathrm{i}}$ at associated times $t_{i}$, it is convenient first to calculate the mean of the measurements and to subtract this from the measurements themselves, to give a set of measurements $y_{i}$ whose mean is now zero. That is to say,

$$
y_{i}=m_{i}-\mu \text { where } \mu=n^{-1} \sum_{i=1}^{n} m_{i}
$$

Likewise it is convenient to subtract the mean of the times from each time to give a set of times $x_{i}$ whose mean is zero,

$$
x_{i}=t_{i}-\lambda \text { where } \lambda=n^{-1} \sum_{i=1}^{n} t_{i}
$$

The French mathematician J. J. Fourier [3] showed that any periodic function (of mean zero and fundamental frequency $2 \pi \omega$ ) may be expressed as a sum of sinusoid terms, a fundamental $(r=1)$ and its harmonics $(r>1)$ :

$$
Z(x)=\sum_{r=1}^{\infty} a_{r} \cos \omega r x+\sum_{r=1}^{\infty} b_{r} \sin \omega r x
$$

where $a_{r}$ and $b_{r}$ are parameters to be determined. Initially it will be assumed that the harmonics are all zero, that is $a_{r}$ and $b_{r}$ will be zero for all $r>1$ so the function $Z(x)$ will be a pure sinusoid. More complicated periodic functions will be discussed later.

$$
\text { Hence } Z(x)=a \cos \omega x+b \sin \omega x=r \sin (\omega x+\varphi)
$$

where $r$ is the semi-amplitude (i.e. half peak-to-peak) and $\varphi$ is the phase at $x=0 ; r=\sqrt{a^{2}+b^{2}}, \varphi=\tan ^{-1} \frac{a}{b}$. The values of $\mathrm{a}$ and $\mathrm{b}$ can be estimated as follows:

$$
a_{\text {est }}=2 n^{-1} \sum_{i=1}^{n} y_{i} \cos \omega x_{i} ; \quad b_{\text {est }}=2 n^{-1} \sum_{i=1}^{n} y_{i} \sin \omega x_{i} .
$$

Hence $r$ and $\varphi$ may be estimated thus:

$$
\begin{gathered}
r_{\text {est }}=\sqrt{a_{\text {est }}^{2}+b_{\text {est }}^{2}} \\
\varphi_{\text {est }}=\tan ^{-1} \frac{a_{\text {est }}}{b_{\text {est }}}
\end{gathered}
$$

The true value of $\omega$ can be estimated as the value of $\omega_{\text {est }}$ which maximises $r_{\text {est, }}$, that is such that $\partial / \partial \omega\left(r_{\text {est }}\right)=0$. The estimated frequency, $f_{\text {est }}$, is then $2 \pi \omega_{\text {est }}$.

The quantity $\omega_{\text {est }}$ can be found in a variety of ways, including the Newton-Raphson method [3], direct algebraic evaluation or the bisection of the ordinate [4]. It is important to realise that, given the same set of $x_{i}$ and $y_{i}$ data, the same $\omega_{\text {est }}$ will be derived whichever method of calculation is adopted. The value of $\omega_{\text {est }}$ will however change whenever $x_{i}$ and $y_{i}$ vary. It can be assumed that $x_{i}$ will be effectively immune from error (the observer's clock will be generally accurate), but any change in an observational datum-if $y_{i}$ becomes $y_{i}+\varepsilon_{i}$, say,-will produce a corresponding change in $\omega_{\text {est }}$. Changes in the observational data will also affect $r_{\text {est }}$ (the estimated semi-amplitude) and $\varphi_{\text {est }}$ (the estimated phase). The following paragraphs show how the effects on the statistics $\omega_{e s t}, r_{e s t}$ and $\varphi_{\text {est }}$ can be calculated from the imputed observational errors.

Suppose now that $e_{i}$ is the probable error in the $i^{\text {th }}$ observation, that is, that $e_{i}$ is the standard deviation of $\varepsilon_{i}$.

\section{Accuracy of Estimated Frequency and Period}

To simplify the calculation that follows define $C(\omega)=\sum_{i=1}^{n} y_{i} \cos \omega x_{i}$ and $S(\omega)=\sum_{i=1}^{n} y_{i} \sin \omega x_{i}$. For a large, randomly-timed set of data $\sum_{i=1}^{n} \cos ^{2} \omega x_{i} \approx n / 2$ and $\sum_{i=1}^{n} \sin ^{2} \omega x_{i} \approx n / 2$, then let $Q^{2}=C^{2}+S^{2}$ where $Q$ $\approx n r_{e s t} / 2$. Write $Q^{\prime}=\mathrm{d} Q / \mathrm{d} \omega, C^{\prime}=\mathrm{d} C / \mathrm{d} \omega$ and $S^{\prime}=\mathrm{d} S / \mathrm{d} \omega$ noting that $C^{\prime}=-\sum_{i=1}^{n} y_{i} x_{i} \sin \omega x_{i}$ and 
$S^{\prime}=\sum_{i=1}^{n} y_{i} x_{i} \cos \omega x_{i}$.

Writing $Q^{\prime \prime}=\mathrm{d}^{2} Q / \mathrm{d} \omega^{2}, C^{\prime \prime}=\mathrm{d}^{2} C / \mathrm{d} \omega^{2}$ and $S^{\prime \prime}=\mathrm{d}^{2} S / \mathrm{d} \omega^{2}$, we have $C^{\prime \prime}=-\sum_{i=1}^{n} y_{i} x_{i}^{2} \cos \omega x_{i}$ and $S^{\prime \prime}=-\sum_{i=1}^{n} y_{i} x_{i}^{2} \sin \omega x_{i}$. These quantities will be used in the calculations that follow.

At the best estimate for $\omega, Q^{\prime}=0$ and

$$
C C^{\prime}+S S^{\prime}=0
$$

Differentiating this with respect to the observation $y_{i}$ gives:

$$
\begin{aligned}
& \left(\partial C / \partial y_{i}+\partial C / \partial \omega \cdot \partial \omega / \partial y_{i}\right) C^{\prime}+\left(\partial C^{\prime} / \partial y_{i}+\partial C^{\prime} / \partial \omega \cdot \partial \omega / \partial y_{i}\right) C \\
& +\left(\partial S / \partial y_{i}+\partial S / \partial \omega \cdot \partial \omega / \partial y_{i}\right) S^{\prime}+\left(\partial S^{\prime} / \partial y_{i}+\partial S^{\prime} / \partial \omega \cdot \partial \omega / \partial y_{i}\right) S=0
\end{aligned}
$$

Gathering the terms according to the derivative, and noting that $\partial C / \partial \omega=C^{\prime}, \partial S / \partial \omega=S^{\prime}, \quad \partial C^{\prime} / \partial \omega=C^{\prime \prime}$, $\partial S^{\prime} / \partial \omega=S^{\prime \prime}$ and $C^{\prime 2}+C C^{\prime \prime}+S^{\prime 2}+S S^{\prime \prime}=Q Q^{\prime \prime}$,

$$
\partial \omega / \partial y_{i}\left(Q Q^{\prime \prime}\right)+C^{\prime} \cos \omega x_{i}-C x_{i} \sin \omega x_{i}+S^{\prime} \sin \omega x_{i}-S x_{i} \cos \omega x_{i}=0
$$

The quantity which interests us is $e_{\omega}$, the probable error in $\omega$. As a function of $e_{i}$, this is given by

$$
e_{\omega}^{2}=\sum_{i=1}^{n} e_{i}^{2}\left(\partial \omega / \partial y_{i}\right)^{2}
$$

If we assume that all the observational errors are all from the same distribution, and this has standard error $e$, substituting (5) into (6) gives:

$$
e_{\omega}=e\left(\left|Q Q^{\prime \prime}\right|\right)^{-1} \sqrt{\sum_{i=1}^{n}\left\{\left(C^{\prime} \cos \omega x_{i}-C x_{i} \sin \omega x_{i}+S^{\prime} \sin \omega x_{i}-S x_{i} \cos \omega x_{i}\right)^{2}\right\}}
$$

For large, randomly-timed sets of data, the cross-terms in the expansion of the above expression become negligible, therefore

$$
e_{\omega}=e\left(\left|Q Q^{\prime \prime}\right|\right)^{-1} \cdot \sqrt{\left\{C^{\prime 2} \sum_{i=1}^{n} \cos ^{2} \omega x_{i}+C^{2} \sum_{i=1}^{n} x_{i}^{2} \sin ^{2} \omega x_{i}+S^{\prime 2} \sum_{i=1}^{n} \sin ^{2} \omega x_{i}+S^{2} \sum_{i=1}^{n} x_{i}^{2} \cos ^{2} \omega x_{i}\right\}} .
$$

Again, for large randomly-timed sets of data, the calculation can be simplified by noting again that $\sum_{i=1}^{n} \cos ^{2} \omega x_{i} \approx n / 2$ and $\sum_{i=1}^{n} \sin ^{2} \omega x_{i} \approx n / 2$. Also, because $\sum_{i=1}^{n} x_{i}=0, \sum_{i=1}^{n} x_{i}^{2} \approx n T^{2} / 12$ where $T$ is the span of time over which observations were made, from first to last. So $\sum_{i=1}^{n} x_{i}^{2} \sin ^{2} \omega x_{i} \approx n T^{2} / 24$ and $\sum_{i=1}^{n} x_{i}^{2} \cos ^{2} \omega x_{i} \approx n T^{2} / 24$. Thus we have

$$
e_{\omega}=e\left(\left|Q Q^{\prime \prime}\right|\right)^{-1} \cdot \sqrt{\left((n / 2)\left\{C^{\prime 2}+S^{\prime 2}+\left(T^{2}\left(C^{2}+S^{2}\right) / 12\right)\right\}\right)}
$$

This can be simplified still further, albeit with some loss of accuracy for smaller data sets, by noting that $C^{\prime}$ and $S^{\prime}$ will be small compared to $S$ and $C$ so that

$$
\left|Q Q^{\prime \prime}\right|=\left|C^{\prime 2}+C C^{\prime \prime}+S^{\prime 2}+S S^{\prime \prime}\right|=\left(a_{\text {est }}^{2}+b_{\text {est }}^{2}\right) \cdot\left(n^{2} / a\right) \cdot\left(T^{2} / 12\right)=r_{\text {est }}^{2} \cdot n^{2} \cdot T^{2} / 48 .
$$

Thus $e_{\omega}=e\left(r_{\text {est }}^{2} \cdot n^{2} \cdot T^{2} / 48\right)^{-1} \cdot \sqrt{\left\{(n / 2) \cdot\left(T^{2} / 12\right) \cdot\left((n / 2) \cdot r_{\text {est }}\right)^{2}\right\}}=e \cdot\left(T \cdot r_{\text {est }} \cdot \sqrt{n}\right)^{-1} \cdot 2 \sqrt{6}$.

In reality, the implicit prior evaluation of the mean magnitude, the phase and the amplitude gives a reduction by 3 of the number of degrees of freedom. Hence $e_{\omega}=e \cdot\left(T \cdot r_{\text {est }} \cdot \sqrt{(n-3)}\right)^{-1} \cdot 2 \sqrt{6}$ is slightly more accurate for small values of $n$.

The corresponding formula for frequency $e_{f}=e_{\omega} /(2 \cdot \pi)=e \cdot\left(\pi T \cdot r_{\text {est }} \sqrt{(n-3)}\right)^{-1} \cdot \sqrt{6}$

And for the period $P=1 / f$ the corresponding formula is $e_{P}=P^{2} e \cdot\left(\pi T \cdot r_{\text {est }} \cdot \sqrt{(n-3)}\right)^{-1} \cdot \sqrt{6}$ 
The dimensional part of the Formula (8) (that is, $e /\left(\operatorname{Tr}_{\text {est }}\right)$ ) agrees with the results given by Kovacs [5] who also derived a $1 / \sqrt{n}$ term for large $n$.

Formulae (8) and (9) are deserving of a few general remarks. The errors in both frequency and period are inversely proportional to $T$, the span of the observations: that is, all other things being equal, making observations for, say, twice as long, will give half the error in period or frequency. Also both errors are proportional to e, the mean observational error, as common sense would dictate. Moreover both are inversely proportional to $r_{\text {est }}$. Hence the accuracy in frequency or period estimation is essentially dictated by the ratio of observational error to oscillatory amplitude. Again this is common sense: when it comes to calculating the period, the bigger the oscillation, the bigger the error one can afford when observing it. The term $1 / \sqrt{n}$ (for large $n$ ) commonly occurs when using (assumed) statistically independent observations to calculate statistical parameters. It suggests a certain "law of diminishing returns", in that, say, 100 times as many observations will only give 10 times the accuracy. If the statistical independence is compromised, the value of each individual observation will be less still. In the extreme case, there is clearly no advantage in repeating the same observation many times (with the same observational error) in a short time interval.

\section{Accuracy of the Estimated Phase}

Starting from (3), and assuming, as before, that $\sum_{i=1}^{n} \cos ^{2} \omega x_{i} \approx n / 2$ and $\sum_{i=1}^{n} \sin ^{2} \omega x_{i} \approx n / 2$, gives $\varphi_{\text {est }}=\tan ^{-1}\left(\frac{C}{S}\right)$.

Differentiating with respect to the observation $y_{i}$ gives:

$$
\begin{aligned}
\mathrm{d} \varphi_{\text {est }} / \mathrm{d} y_{i}= & \partial \varphi_{e s t} / \partial y_{i}+\partial \varphi_{\text {est }} / \partial \omega \cdot \partial \omega / \partial y_{i} . \\
= & \left(Q^{3} Q^{\prime \prime}\right)^{-1} \cdot\left\{S\left(S S^{\prime \prime}+C C^{\prime \prime}\right) \cos \omega x_{i}-C\left(S S^{\prime \prime}+C C^{\prime \prime}\right) \sin \omega x_{i}\right. \\
& \left.-Q^{2} C^{\prime} x_{i} \cos \omega x_{i}-Q^{2} S^{\prime} x_{i} \sin \omega x_{i}\right\} .
\end{aligned}
$$

By analogy with Equation (6),

$$
e_{\varphi}^{2}=\sum_{i=1}^{n} e_{i}^{2}\left(\mathrm{~d} \varphi_{\text {est }} / \mathrm{d} y_{i}\right)^{2}
$$

Simplifying by removing the negligible terms as before gives

$$
e_{\varphi}=e\left(Q^{2} \cdot\left|Q^{\prime \prime}\right|\right)^{-1} \cdot \sqrt{(n / 2) \cdot\left\{\left(S S^{\prime \prime}+C C^{\prime \prime}\right)^{2}+T^{2} Q^{2}\left(C^{\prime 2}+S^{\prime 2}\right) / 12\right\}}
$$

With the usual substitutions for $Q, Q^{\prime \prime}, S, C, S^{\prime}, C^{\prime}, S^{\prime \prime}$ and $C^{\prime \prime}$ this simplifies to

$$
e_{\varphi}=\left(e / r_{\text {est }}\right) \cdot \sqrt{(2 /(n-2))}
$$

The $n-2$ term signifies the implicit loss of 2 degrees of freedom, namely the mean value and the amplitude of the oscillation.

\section{Accuracy of the Estimated Amplitude}

Beginning from Equation (5) and again assuming that $\sum_{i=1}^{n} \cos ^{2} \omega x_{i} \approx n / 2$ and $\sum_{i=1}^{n} \sin ^{2} \omega x_{i} \approx n / 2$, gives $r_{\text {est }}$ $=2 Q / n$. Differentiating with respect to the observation $y_{i}$ gives:

$$
\mathrm{d} r_{\text {est }} / \mathrm{d} y_{i}=\partial r_{\text {est }} / \partial y_{i}+\partial r_{\text {est }} / \partial \omega \cdot \partial \omega / \partial y_{i}=(2 /(n Q)) \cdot\left(S \sin \omega x_{i}+C \cos \omega x_{i}\right) .
$$

Again by analogy with Equation (6), $e_{r}^{2}=\sum_{i=1}^{n} e_{i}^{2}\left(\mathrm{~d} r_{\text {est }} / \mathrm{d} y_{i}\right)^{2}$

Simplifying by removing the negligible terms as before gives

$$
e_{r}=e \cdot \sqrt{(2 /(n-1))}
$$

where the $n-1$ term signifies the implicit loss of one degree of freedom, namely the mean value. 


\section{Discussion: Non-Sinusoidal Periodic Data, and Data Having Multiple Periodicities}

As mentioned earlier, any periodic function may be expressed as a sum of a series of harmonic terms (see Equation (1)). Since the frequency of each term is an exact multiple of the fundamental, it would be expected that the cross-terms in the above formulae would all vanish for any harmonic, so they will not affect the veracity of the Equations (9), (10), (13) and (15). This is indeed the case and will be shown later by Monte-Carlo methods. Even when frequencies are present that are not exact multiples of the fundamental—as with SR variables, for example, when frequencies are commonly present in the ratios of about 1.5 - 2.0 and 10 - 15 [6] - the cross-terms are still likely to vanish; particularly if the data spans many cycles of all the frequencies that are present, so that the estimate of the fundamental frequency will not suffer greatly whatever the interfering frequencies might be. This too will be supported by the Monte-Carlo analysis to follow. Mattei et al. [7] give many examples of ratios in the range 1.7 - 1.95 which lie in the first category above. If the interfering signal is itself continuously variable in frequency, or chaotic, then a spread of frequencies is likely to be involved, and in such cases it will generally be necessary to regard these as random noise which contributes to the term $e$; in other words, for the purpose of statistical error estimations, they can be handled in the same way as extra observational errors. In the extreme case, e can be set equal to the standard deviation of the entire data set so that even the "signal" is regarded as part of the noise. When the signal is submerged in the noise, the error in using this value for e will be tiny, and will lead to slight overestimates of the errors in the statistics, rather than underestimates.

As well as furnishing a ready means to compare statistics derived from observations with their catalogued equivalents, the methods described herein enable one to say whether the period of variation (or phase or amplitude) is changing with time. It is necessary simply to divide the observations into batches, each covering a different time interval, then to calculate and compare the statistics from each batch. Significant changes can show that the system is evolving or that it is slowing down or speeding up, perhaps due to energy being radiated away or to the system slowly collapsing.

\section{Monte-Carlo Runs}

Monte-Carlo is a powerful mathematical technique [8] that can be used to derive results where closed formulae either do not exist or are too complicated to use. Apart from simple "hit-and-miss" applications, one or all of the input parameters are generally only estimatable by their probability distribution. Monte-Carlo analysis consists of running a mathematical algorithm (a sequence of equations) many times (often thousands) with a different set of input parameters on each occasion, as described by their probability distribution. The multitude of different outcomes are can then be compared and listed, and probabilities calculated for each possible outcome. The validation of the formulae that were derived earlier lends itself to a Monte-Carlo process: the input parameters are the set of synthetic data (constant and known) and the accompanying synthetic observational errors (variable, drawn from a statistically normal distribution). The outcomes are the estimates of the frequency, amplitude and phase, and it is these which are to be compared with the values given by the formulae in Equations (9), (13) and (15) above.

Initially the synthetic data shall comprise a single periodicity without harmonics, with varying degrees of noise superimposed. The underlying mean of the data shall be zero, the semi-amplitude r (see Equation (2)) shall be fixed as 1.0, so the variation will go from +1 to -1 before the errors are applied. These values will often be in stellar magnitude, but need not be in general. The phase, $\varphi$, is measured in radians and shall be taken as zero and the frequency shall be 1 unit, giving $\omega=2 \pi$. The time span shall run from -5 to +5 units, hence $T$, the span of time over which the synthetic observations were deemed to have been made, is 10 units, giving precisely 10 complete oscillations. A huge range of time spans is, of course, possible, depending on the unit chosen, ranging from years (a Mira variable) to seconds (a pulsar)! Three distinct values of observational error were compared: (i) 0.01, (ii) 0.1 and (iii) 1.0 units, with 1000 evenly spaced times chosen to cover the entire 10 unit time span. In practice, if a binning process is used then the observations, could, indeed, be equally spaced. Even if the times were randomly spaced, the same general trends would be observed, except that aliasing effects would potentially be present if there were serious time gaps in the data. These must be considered separately and are beyond the scope of the present study. In each of the sets (i) - (iii), 1000 Monte-Carlo runs were considered and the parameters $f, \varphi$ and $r$ were estimated for each run. When all the runs had been done, the standard deviations for these 
parameters $e_{f}, e_{\varphi}, e_{r}$, were calculated to compare with the theoretical values given in Equations (9), (11) and (12).

The results are shown below in Table 1 . For illustration, Figure 1 shows a typical data from set (iii) (one of the 1,000 runs) with a standard observational error of 1.0 .

It can be seen from Table 1 that there is excellent agreement between the Monte-Carlo average values of each statistic (c) and the corresponding true value (b). This shows that the estimates obtained are essentially unbiased. Furthermore the standard errors obtained from the formulae (d) show excellent agreement with the corresponding Monte-Carlo values (e), which serves to verify the formulae in this case.

Two further sets of Monte-Carlo runs, denoted in Table 2 by (iv) and (v), were performed in which a further periodicity was added to the $e=1.0$ case. Set (iv) had an additional periodicity of frequency 2 units, of semi-amplitude 0.5 and phase $\pi / 2$ radians. As before, 1,000 Monte-Carlo replications were performed. For illustration, Figure 2 shows a typical set of data with the underlying light curve (before the errors were applied) picked out in a solid line. Its periodic, but non-sinusoidal nature is readily discerned. An RV Tauri-type variable star, with its characteristically alternating deep and shallow minima, could be approximated by this data set.

Set (v) had the same parameters as (iv) except that the added frequency was now 2.7183 units (the base of natural logarithms) which, not being a rational number, is not an integral or rational ratio to the fundamental. (Any irrational number, for example $\sqrt{2}$ or $\sqrt{3}$, would have sufficed as the added frequency). Again, 1000 Monte-Carlo replications were performed. For illustration, Figure 3 shows a typical set of data with the underlying light curve (before the errors were applied) picked out in a solid line. This time the light curve beneath the noise does not repeat exactly after any finite period.

From Table 2 it can be seen that there is good agreement between the Monte-Carlo average values of each statistic (c) and the corresponding true value (b), though now the presence of additional frequencies skews some of the averages very slightly. The standard error obtained from the formulae (d) again show excellent agreement with the corresponding Monte-Carlo values (e), which demonstrates that harmonics of the fundamental and, indeed, arbitrary periodic components have little effect on the accuracy of the derived statistics relating to the fundamental itself.

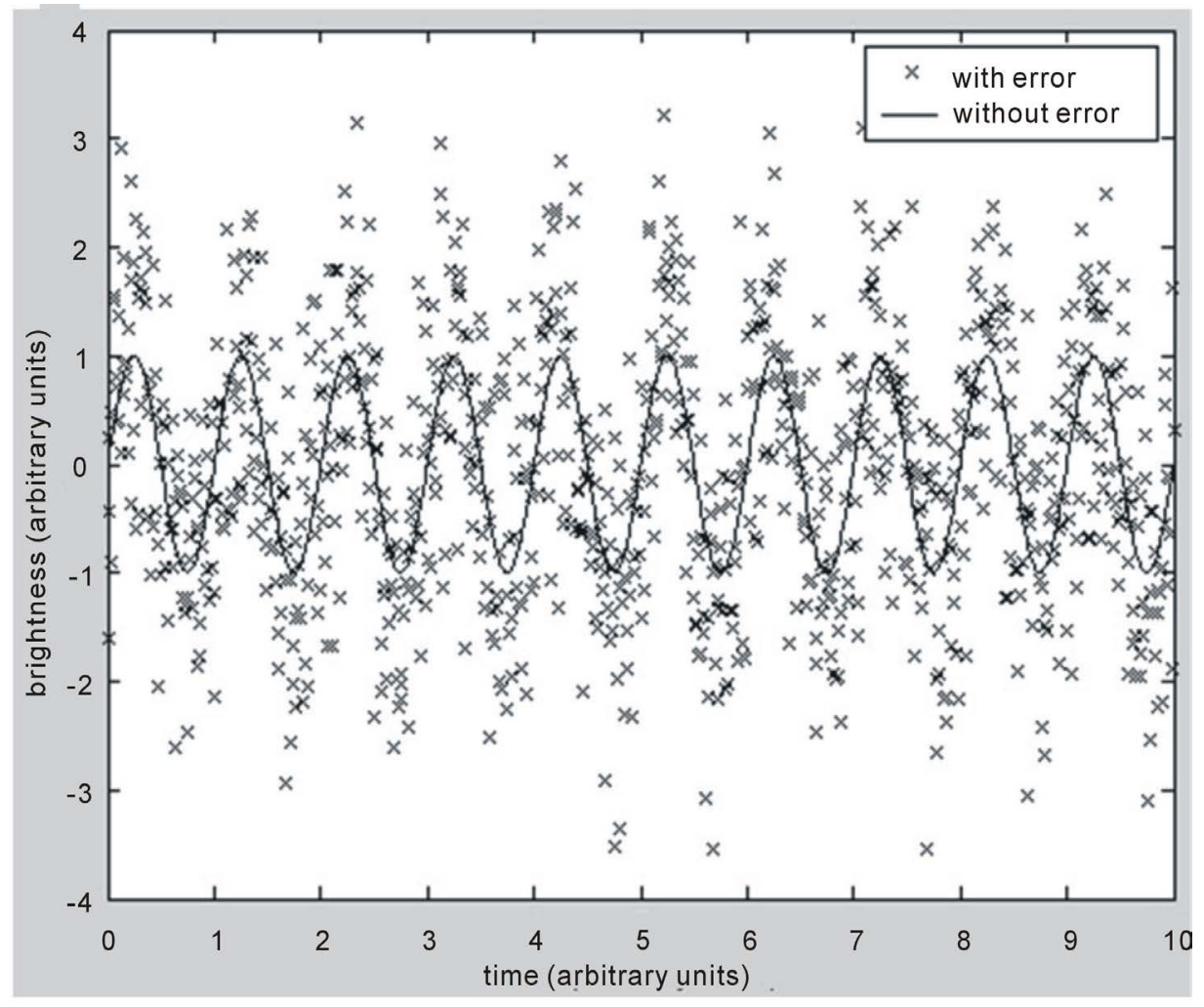

Figure 1. Sample Monte-Carlo run with standard error = 1.0. 
Table 1. Comparison of Monte-Carlo Statistics with the theoretical values.

\begin{tabular}{cccccc}
\hline \multirow{2}{*}{ Standard deviation of noise } & $\begin{array}{c}\text { (a) } \\
\text { Statistic }\end{array}$ & $\begin{array}{c}\text { (b) } \\
\text { True value }\end{array}$ & $\begin{array}{c}\text { (c) } \\
\text { Monte-Carlo average }\end{array}$ & $\begin{array}{c}\text { (d) } \\
\text { Formulaic standard } \\
\text { error }\end{array}$ & $\begin{array}{c}\text { Monte-Carlo derived } \\
\text { standard error }\end{array}$ \\
\hline \multirow{2}{*}{ (i) $e=0.01$} & $f$ & 1.0 & 1.0000 & 0.0000247 & 0.0000246 \\
& $\varphi$ & 0.0 & 0.0000 & 0.000447 & 0.000436 \\
(ii) $e=0.1$ & $r$ & 1.0 & 1.0000 & 0.000447 & 0.000448 \\
& $f$ & 1.0 & 1.0000 & 0.000247 & 0.000246 \\
(iii) $e=1.0$ & $\varphi$ & 0.0 & 0.0002 & 0.00447 & 0.00437 \\
& $r$ & 1.0 & 1.0002 & 0.00447 & 0.00447 \\
& $f$ & 1.0 & 1.0002 & 0.00247 & 0.00247 \\
& $\varphi$ & 0.0 & 0.0020 & 0.0447 & 0.0439 \\
\hline
\end{tabular}

Table 2. Comparison of Monte-Carlo Statistics with the theoretical values with added periodicities present.

\begin{tabular}{|c|c|c|c|c|c|}
\hline $\begin{array}{l}\text { Standard deviation of } \\
\text { noise is } e=1.0\end{array}$ & $\begin{array}{c}\text { (a) } \\
\text { Statistic }\end{array}$ & $\begin{array}{c}\text { (b) } \\
\text { True value }\end{array}$ & $\begin{array}{c}\text { (c) } \\
\text { Monte-Carlo average }\end{array}$ & $\begin{array}{l}\text { (d) } \\
\text { Formulaic standard } \\
\text { error }\end{array}$ & $\begin{array}{l}\text { (e) } \\
\text { Monte-Carlo derived } \\
\text { standard error }\end{array}$ \\
\hline \multirow{3}{*}{$\begin{array}{l}\text { (iv) added frequency of } 2 \text { units } \\
\text { (semi-amplitude }=0.5, \\
\text { phase }=\pi / 2 \text { ) }\end{array}$} & $f$ & 1.0 & 1.0000 & 0.00247 & 0.00247 \\
\hline & $\varphi$ & 0.0 & 0.00296 & 0.0447 & 0.0438 \\
\hline & $r$ & 1.0 & 1.0039 & 0.0447 & 0.0447 \\
\hline \multirow{3}{*}{$\begin{array}{c}\text { (v) added frequency of } 2.7183 \\
\text { units }(\text { semi-amplitude }=0.5 \text {, } \\
\text { phase }=\pi / 2)\end{array}$} & $f$ & 1.0 & 1.0000 & 0.00247 & 0.00247 \\
\hline & $\varphi$ & 0.0 & -0.00618 & 0.0447 & 0.0439 \\
\hline & $r$ & 1.0 & 1.0039 & 0.0447 & 0.0447 \\
\hline
\end{tabular}

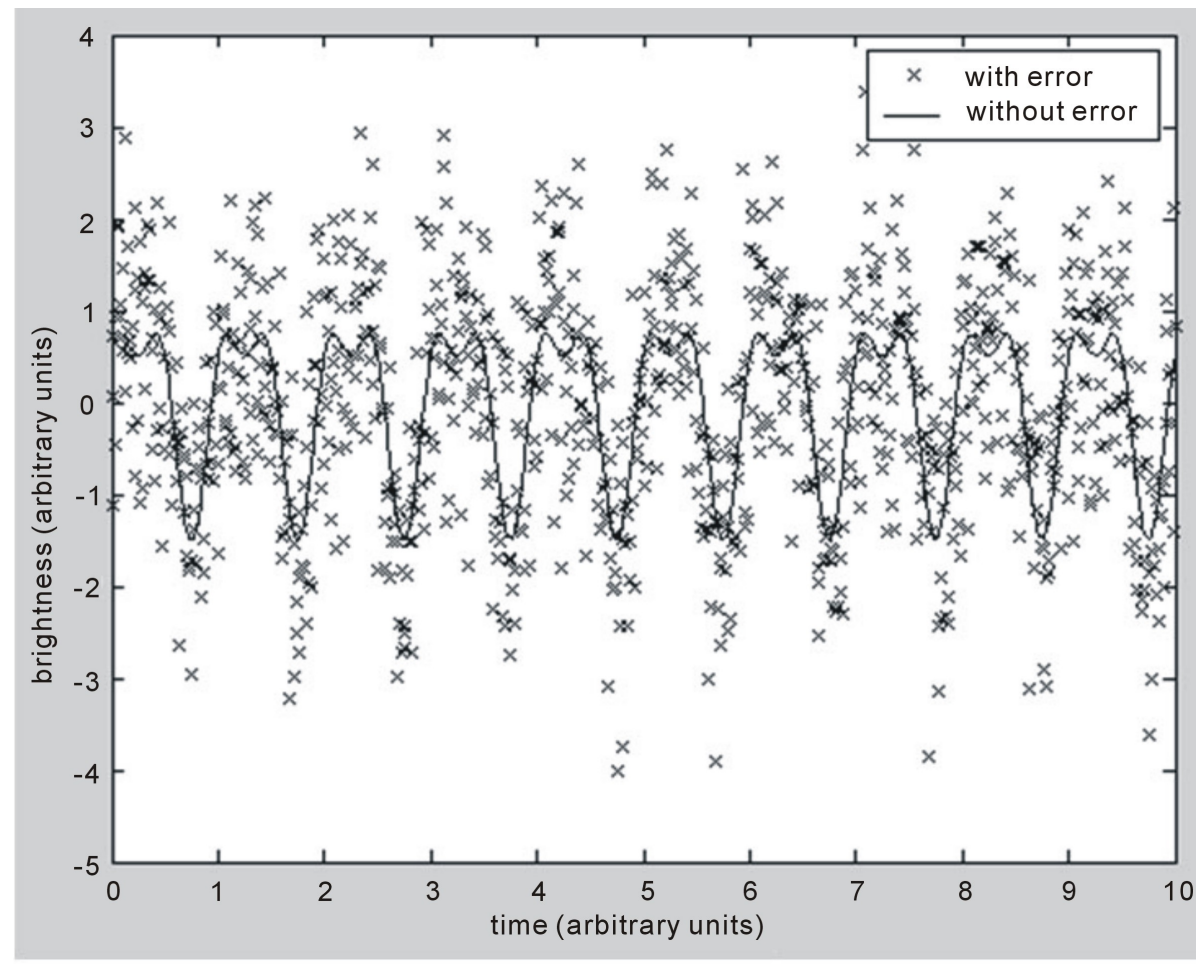

Figure 2. Sample Monte-Carlo run with standard error = 1.0 and added rational frequency = 2 . 


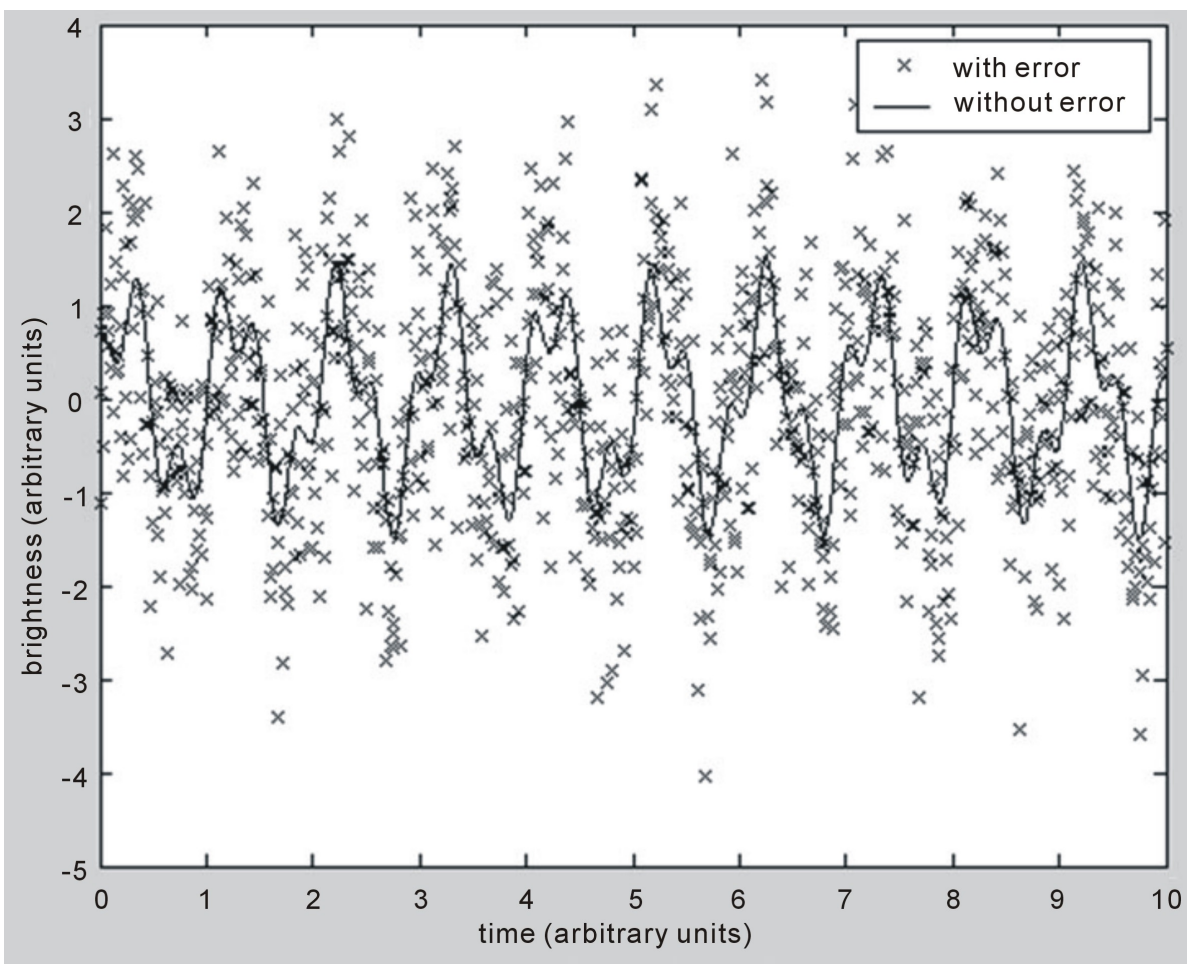

Figure 3. Sample Monte-Carlo run with standard error $=1.0$ and added irrational frequency $=$ 2.7183 .

\section{Summary of Conclusions}

This paper has shown that, given very general assumptions concerning a set of periodic data, it is possible to calculate, as a function of observational accuracy, likely errors for the derived period, its phase and its amplitude. If several sets of data have been collected each covering a different era, the statistics may be used to determine whether the period, phase or amplitude are changing with time. Such changes can be symptomatic of energy loss or evolution. The validity of such methods has been clearly shown using sets of simulated (Monte-Carlo) data.

\section{References}

[1] Howarth, J.J. and Greaves, J. (2001) Amplitude and Phase Changes in the Light Curves of Long-Period Variables. Monthly Notices of the Royal Astronomical Society, 325, 1383-1388. http://dx.doi.org/10.1046/j.1365-8711.2001.04489.x

[2] Koen, C. (1990) Significance Testing of Periodogram Ordinates. Astrophysical Journal, 348, 700. http://dx.doi.org/10.1086/168277

[3] Margenau, H. and Murphy, G.M. (1968) The Mathematics of Physics and Chemistry. Van Nostrand Reinhold, New York.

[4] Cohen, A.M. (1973) Numerical Analysis. McGraw-Hill, New York.

[5] Kovacs, G. (1981) Frequncy Shift in Fourier Analysis. Astrophysics and Space Science, 78, 175-188. http://dx.doi.org/10.1007/BF00654032

[6] Percy, J.R. and Polano, S. (1998) Pulsation Modes in M Giants. In: Bradley, P.A. and Guzik, J.A., Eds., A Half-Century of Stellar Pulsation Interpretations: A Tribute to Arthur N Cox, Astronomical Society of the Pacific Conference Series, 135, 249.

[7] Mattei, J.A., Foster, G., Hurwitz, L.A., Malatesta, K.H., Willson, L.A. and Mennessier, M.-O. (1997) Classification of Red Variables. In: Battrick, B., Ed., Proceedings of the ESA (European Space Agency) Symposium, ESA, Noordwijk, ESA SP-402, 269.

[8] Hammersley, J.M. and Handscomb, D.C. (1964) Monte-Carlo Methods. Springer Netherlands http://dx.doi.org/10.1007/978-94-009-5819-7 